\title{
Article
}

\section{Long lasting reversibility of the mitochondrial permeability transition in Saccharomyces cerevisiae.}

\author{
Lilia Morales-García ${ }^{1,2,3}$, Carolina Ricardez-García ${ }^{1,3}$ Paulina Castañeda-Tamez ${ }^{1}$, Natalia Chiquete-Félix ${ }^{1}$ and Sal- \\ vador Uribe-Carvajal ${ }^{1,2 *}$ \\ 1 Department of Genetics and Molecular Biology, Instituto de Fisiología Celular, UNAM, Mexico City, Mexico; \\ rcoria@ifc.unam.mx \\ 2 Department of Biochemistry, Medicine School, UNAM, Mexico City, Mexico; fedebioquimica@bq.unam.mx. \\ 3 Both authors are first authors as they contributed equally to the project. \\ * Correspondence: suribe@ifc.unam.mx; Tel.:5555625632
}

\begin{abstract}
The Saccharomyces cerevisiae mitochondrial unspecific pore ( $s c \mathrm{MUC}$ ) is an uncoupling unspecific pore that shares some similarities with the mammalian permeability transition pore (mPTP). When open, both channels deplete ion and proton gradients across the inner mitochondrial membrane. However, the role of $\mathrm{mPTP}$ is to reversibly open to protect cells against stress. If $\mathrm{mPTP}$ remains stuck in the open position the cell dies. In contrast, scMUC is probably dedicated to deplete oxygen from the medium in order to kill competing organisms. Such $\mathrm{O}_{2}$ depletion would be better achieved if oxidative phosphorylation is at least mildly uncoupled. Still, when oxidative phosphorylation is needed $s_{c}$ MUC should be able to close. To test this, the reversible opening and closing of $s_{c} \mathrm{MUC}$ in the presence of different effectors was tested in isolated mitochondria from S. cerevisiae. Evaluations were conducted at different incubation times, monitoring the rate of $\mathrm{O}_{2}$ consumption, mitochondrial swelling and the transmembrane potential. It was observed that ${ }_{s c}$ MUC did remain reversibly open for minutes. A low energy charge (ATP/ADP) closed the channel. In addition, high $\mathrm{Ca}^{2+}$ promoted closing and it was a highly powerful effector.
\end{abstract}

Keywords: Physiological uncoupling, Yeast mitochondria, Mitochondrial Permeability transition, scMUC, Reversibility.

\section{Introduction}

Mitochondrial oxidative phosphorylation (OxPhos) involves two coupled processes. First, the exergonic transit of electrons through the redox complexes in the respiratory chain (RC) drives an active transport of protons across the inner mitochondrial membrane to generate a $\Delta \mathrm{pH}$ [1]. Then the proton gradient is dissipated by the $\mathrm{F}_{1} \mathrm{~F}_{0}-\mathrm{ATP}$ synthase to produce ATP [1,2]. In the absence of ATP synthesis, $\Delta \mathrm{pH}$ remains high, decreasing the rate of electron flow [3]. Stalled electrons form free radicals in the different redox centers located in $\mathrm{RC}$ complexes that may react spontaneously with $\mathrm{O}_{2}$ to produce highly motile Reactive Oxygen Species (ROS) [4]. While a small amount of ROS is always produced, an excess of ROS may damage proteins and lipids, leading to aging and even cell death $[5,6]$

Increasing the rate of electron flow through the respiratory chain decreases ROS production [7]. In the absence of ATP synthesis, the rate of electron flow may be increased by physiological uncoupling [8]. Physiological uncoupling mechanisms include proton sinks, which may be either non-specific or specific for protons [9] and branched respiratory chains, which express alternative redox enzyme that are not sensitive to the 
$\Delta \mathrm{pH}[8,10]$. Uncoupling must be tightly controlled through differential expression and/or activity regulation in order to avoid depleting cellular ATP [11].

Saccharomyces cerevisiae is a unicellular eukaryote that displays two strategies to manipulate its environment, which are meant to eliminate other organisms that compete for nutrients: A P-type $\mathrm{H}^{+}$-ATPase located in the plasma membrane lowers the external $\mathrm{pH}$ to values around 3.0 [12] and in addition its mitochondrial respiratory chain (RC) depletes $\mathrm{O}_{2}$ from the medium[13]. In these conditions, $S$. cerevisiae cells survives using an anaerobic fermentative metabolism. Thus, mitochondria from $S$. cerevisiae must consume $\mathrm{O}_{2}$ and oxidize $\mathrm{NADH}+\mathrm{H}^{+}$at high rates, independently of whether they phosphorylate ATP or not $[14,15]$. To do this, these mitochondria produce less $\mathrm{ATP} / \mathrm{O}_{2}$ and in addition, a physiological oxidative phosphorylation (OxPhos) uncoupling system is used. Low $\mathrm{ATP} / \mathrm{O}_{2}$ results from the fact that $S$. cerevisiae mitochondria do not have Complex I, instead they have three type two NADH dehydrogenases, which are not proton pumps [16]. This already leads to a high rate of $\mathrm{O}_{2}$ consumption. In addition, the $S$. cerevisiae Mitochondrial Unspecific Channel ( $s$ MUC) may open to uncouple OxPhos. This channel is a proton sink, as it allows the passage of molecules up to $1.1 \mathrm{kDa}$ and depletes all ion gradients, $\Delta \mathrm{pH}$ included [17-19].

The mammalian permeability transition pore (mPTP) is an uncoupling system similar to $s_{c}$ MUC[20]. Both $s_{c} \mathrm{MUC}$ and $\mathrm{mPTP}$ are highly effective to equilibrate proton and ion concentrations between the mitochondrial matrix and the cytoplasm [21]. An open scMUC allows the passage of molecules up to $1.1 \mathrm{kDa}$, while mPTP has a cutoff limit of 1.5 $\mathrm{kDa}[17,22]$. In spite of their functional similarities, the physiological role of each of these channels seems to be different as MPTP opens to protect the cell against ischemia [23], while scMUC is probably designed to deplete $\mathrm{O}_{2}$ [24]. Also, these channels express different sensitivity to some effectors [25]. Notably, $\mathrm{Ca}^{2+}$ closes scMUC while it opens mPTP. In addition, high Pi closes $s_{c}$ MUC but opens mPTP $[18,26]$. Also, ADP closes both channels, but ATP opens scMUC while it closes mPTP [27-30]

An important feature of physiological uncoupling systems is that they have to be tightly controlled to avoid depleting cellular ATP [31]. Unicellular organisms and some tissues such as cardiac muscle, where mitochondria are often exposed to large variations in metabolic demand or $\mathrm{O}_{2}$ and solute concentrations, seem to undergo frequent reversible opening and closing of PTP. However, after long incubation times, the pore may be stuck in the open position, leading to cell death [32-34]. Thus, it was decided to evaluate for how long scMUC remains reversible and to determine which important effectors, such as adenine nucleotides of $\mathrm{Ca}^{2+}$ continue to control opening and closure of this channel after long incubation times.

\section{Results}

\subsection{Effects of ADP/ATP on scMUC.}

Both scMUC and MPTP are considered equivalent, except for some differences in effectors, e.g., in contrast to PTP, both $\mathrm{Ca}^{2+}$ and Pi close scMUC. Adenine nucleotides are among known effectors of both MPTP and scMUC: in MPTP Pi opens the channel while ATP and ADP close it. in yeast ATP opens scMUC, while ADP and Pi close them[3437]Thus, to test scMUC reversibility we alternatively added ATP and ADP in the presence of different Pi concentrations and tested the ability of these molecules to counteract the effects of each other (Fig. 1). In agreement with data in the literature, a slow rate of oxygen consumption was observed at $2.0 \mathrm{mM}$ Pi (Fig. 1-A, trace a), suggesting that scMUC was closed[18,19]. Under these conditions, the addition of different concentrations of ATP increased the rate of oxygen consumption (Fig. 1-A, traces b to e). In contrast, at 0.1 $\mathrm{mM}$ Pi the rate of oxygen consumption was high (Fig. 1-B trace a), suggesting that scMUC was open[19,38]. Here, addition of increasing concentrations of ADP inhibited the rate of 
oxygen consumption, suggesting that ADP closed scMUC, i.e., reverted the open state promoted by low Pi (Fig. 1B traces b to e).

Figure 1. Rates of $\mathrm{O}_{2}$ consumption of isolated yeast mitochondria

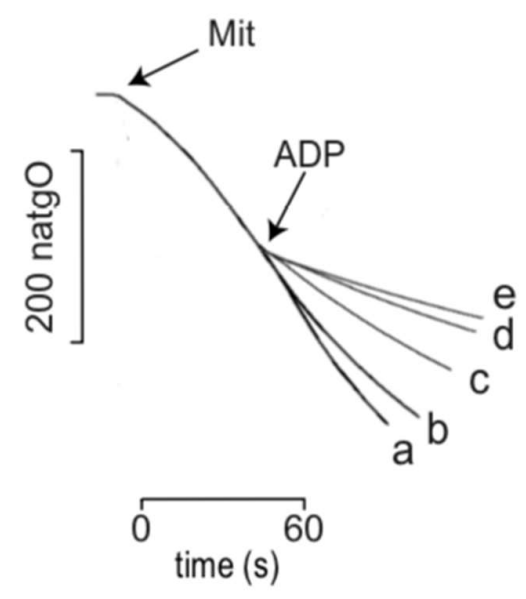

(A)

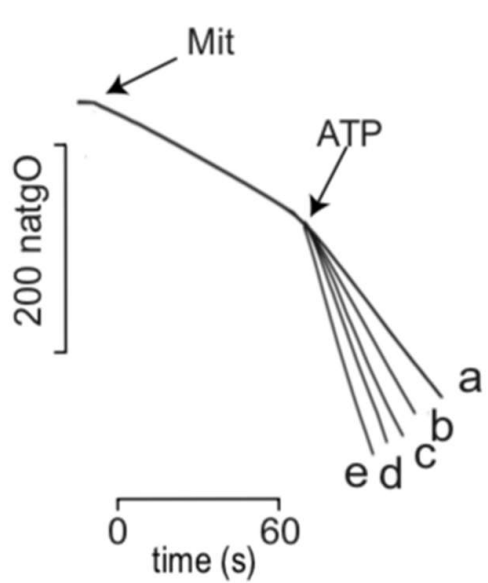

(B)

Figure 1. $\mathrm{O}_{2}$ consumption rates of isolated yeast mitochondria. Effects of phosphate (Pi) ATP and ADP. A: Reaction mixture: $0.6 \mathrm{M}$ mannitol, $5 \mathrm{mM}$ MES , $\mathrm{pH} 6.8,0.1 \mathrm{mM} \mathrm{KCl}, 0.5 \mathrm{mM} \mathrm{MgCl} 2,2 \mu \mathrm{L} / \mathrm{mL}$ ethanol, $0.1 \mathrm{mM}$ phosphate. ADP additions were: a) $2 \mathrm{mM}$; b) $1.5 \mathrm{mM}$; c) $1 \mathrm{mM}$; d) $0.5 \mathrm{mM}$ and e) none ADP. B: Reaction mixture: as in A, except 2 $\mathrm{mM}$ phosphate. ATP additions were: a) none ATP; b) $0.5 \mathrm{mM}$; c) $1 \mathrm{mM}$; d) $1.5 \mathrm{mM}$ and e) $2 \mathrm{mM}$

Oxygen consumption results confirmed that scMUC was regulated by Pi, ADP and ATP. To further explore this phenomenon, it was decided to determine whether changes on $\Delta \Psi$ were consistent with alternating $\mathrm{PT}$ states in response to sequential additions of ADP and ATP (Fig. 2). At low Pi, scMUC was open (Fig. 2A trace a) and $\Delta \Psi$ was low; here, addition of $2 \mathrm{mM}$ ADP closed scMUC increasing $\Delta \Psi$ (Fig. 2-A trace b). Then the ADP-mediated increase in $\Delta \Psi$ was reversed by different [ATP], leading to a second depletion of $\Delta \Psi$ (Fig. 2A, traces c, d,e ). The opposite experiment was also performed: when in the presence of high Pi, scMUC was closed as evidenced by a high $\Delta \Psi$ (Fig. 2B). Here ATP addition led to opening of scMUC decreasing $\Delta \Psi$; (Fig. 2B, trace b). Then the addition of different [ADP] promoted a new rise in $\triangle \Psi$, suggesting scMUC closed again (Fig. 2B traces $c, d, e)$. Thus, the alternative addition of ADP and ATP resulted in a reversible sequence of opening/closing of scMUC (Fig. 2).

Figure 2. Effect on the mitochondrial transmembrane potential. 


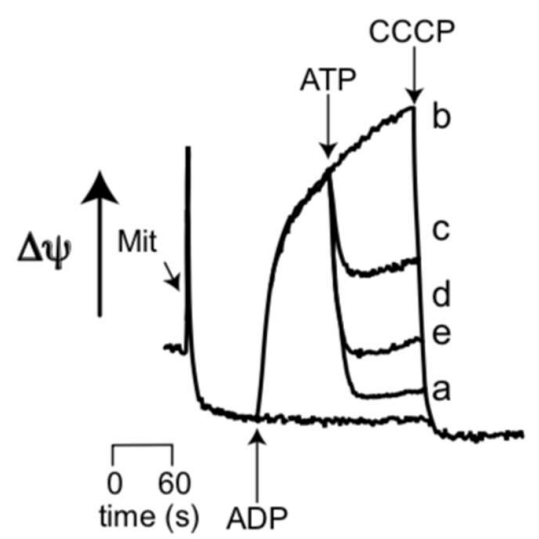

(A)

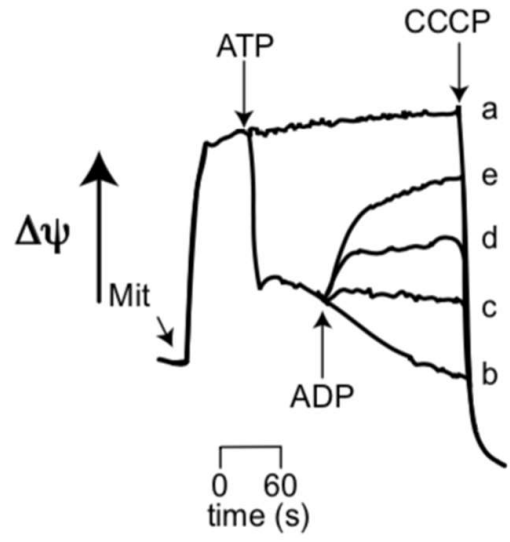

(B)

Figure 2. Effect of the sequential addition of ATP and ADP on the mitochondrial transmembrane potential $(\Delta \Psi)$. Reaction mixture as in Fig. 1, except $15 \mu \mathrm{M}$ safranine-O and Pi. Mit: 0.5mg/mL mitochondria; $0.1 \mathrm{mM}$ Pi (a-e) and $1 \mathrm{mM}$ ADP (b-e) . ATP additions were: a) $0 \mathrm{mM}$; b) $0.5 \mathrm{mM}$; c) $1 \mathrm{mM} \mathrm{d}$ ) $1.5 \mathrm{mM}$; e) 2mM. B 2 mM Pi (a-e) and $1 \mathrm{mM}$ ATP (b-e). ADP additions were: a) $0 \mathrm{mM}$; b) $0.5 \mathrm{mM}$; c) $1 \mathrm{mM}$ d) $1.5 \mathrm{mM}$; e) $2 \mathrm{mM}$. Measurements were conducted in a Ollis/Aminco spectrophotometer in Dual mode at 511-533nm.

Another parameter commonly used to follow PT is mitochondrial swelling. At high $\mathrm{Pi}$ or ADP scMUC was closed and the rate of swelling was slow (Fig. 3, traces a) At low Pi or high ATP, scMUC was open and added $\mathrm{K}^{+}$ion entered the mitochondrial matrix causing a rapid rate of swelling (Fig. 3, traces b) [39]. Alternation between opening and closing was followed. In the presence of $2.0 \mathrm{mM}$ Pi and $1 \mathrm{mM}$ ADP, swelling was slow (Fig. $3 \mathrm{~A}$, trace a) and then, addition of different [ATP] caused increasing rates of swelling, suggesting opening of scMUC (Fig. 3A traces $\mathrm{c}, \mathrm{d}$ and e). In the opposite sense, at 0.1 $\mathrm{mM}$ Pi plus $1 \mathrm{mM}$ ATP a rapid rate of swelling was observed (Fig. 3B, trace b), then, addition of different [ADP] inhibited the rate of swelling (Fig. 3-B traces $c$, d, and e) suggesting that ADP closed scMUC. Therefore, the effect of Pi, ADP or ATP was known in isolation, with these results we observed that the phenomenon of these three effectors; in the same experiment, can open and close making the scMUC dynamic.

Figure 3. . Effect on mitochondrial swelling.

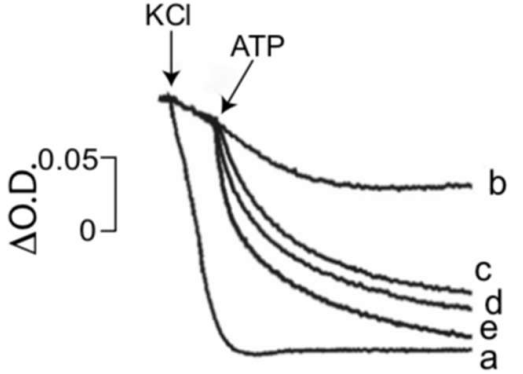

0 time (s) 60

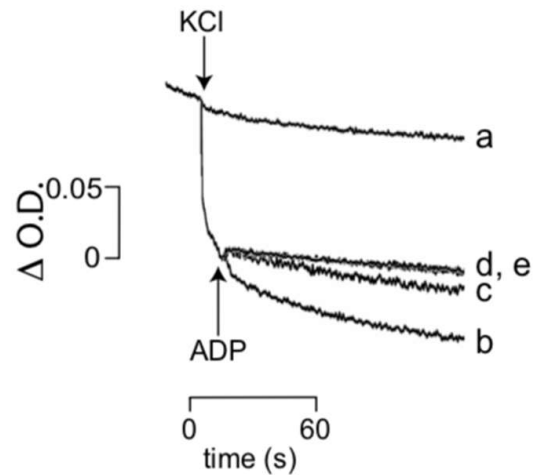


(B)

Figure 3. Effect of the sequential addition of ATP and ADP on mitochondrial swelling. A: Reaction mixture, as in Fig. 1, except $0.3 \mathrm{M}$ mannitol. The arrow indicates the addition of $0.1 \mathrm{M} \mathrm{KCl}$. Swelling was measured in an Ollis/Aminco spectrophotometer in Split mode at $560 \mathrm{~nm} .0 .1 \mathrm{mM}$ Pi (a-e); $1 \mathrm{mM}$ ADP(b-e) . ATP : a) $0 \mathrm{mM}$; b) $0.5 \mathrm{mM}$; c) $1 \mathrm{mM}$; d) $1.5 \mathrm{mM}$; e) 2mM B: as in Fig. 2 except $2 \mathrm{mM}$ Pi. Additions were (a-e); $1 \mathrm{mM}$ ATP (b-e). ADP: a) $0 \mathrm{mM}$; b) $0.5 \mathrm{mM}$; $)$ $1 \mathrm{mM}$; d) $1.5 \mathrm{mM}$; e) $2 \mathrm{mM}$

To test the reversibility of scMUC after long incubation times, yeast mitochondria were incubated for several minutes under conditions where the pore was open and then ADP was added. Both the $\Delta \Psi$ and mitochondrial swelling were monitored. When ADP was added after different incubation times, it was observed that the $\Delta \Psi$ recovered and swelling was reverted suggesting that the scMUC closed, and thus its reversibility was quite robust. (FIG $4 \mathrm{~A} \& \mathrm{~B}$ )

Figure 4. Effect of the addition of ADP on the mitochondrial transmembrane potential and mitochondrial swelling

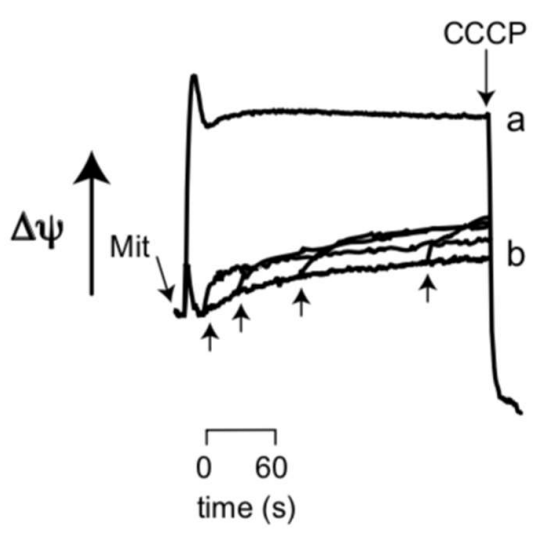

(A)

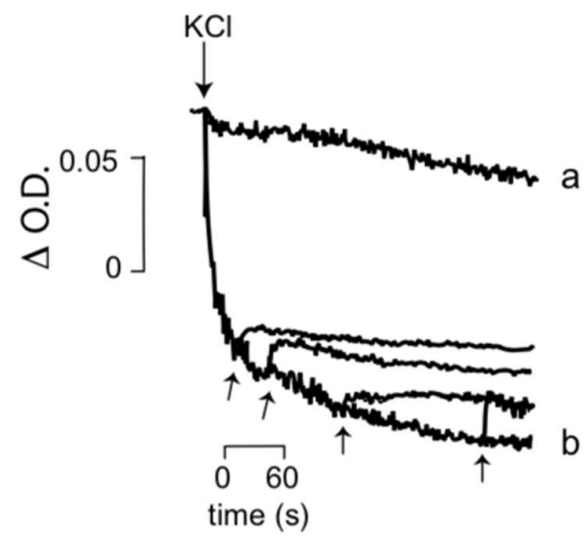

(B)

Figure 4. Effect of the addition of ADP on the mitochondrial transmembrane potential and mitochondrial swelling. Reaction mixture as in Fig. 2 excepts $2 \mathrm{mM}$ ADP were added at different times: 30 seconds, 1, 2 and 4 minutes (upward arrows). B: Reaction mixture, as in Fig. 3. The arrow indicates the addition of $0.1 \mathrm{M} \mathrm{KCl}$. Additions of $2 \mathrm{mM}$ ADP were at different times: 30 seconds, 1, 2 and 4 minutes (arrows upward).

The above results indicate that the opening response of scMUC to the sequential additions of ATP and ADP is reversible for several minutes of incubation. The next question addressed here is whether $s c \mathrm{MUC}$ reversibility is observed in the presence of other effectors, so we decided to test the effect of $\mathrm{Ca}^{2+}$ and EGTA at different incubation times.

\subsection{Effects of $\mathrm{Ca}^{2+} / \mathrm{EGTA}$ additions on scMUC.}

$\mathrm{Ca}^{2+}$ withdrawal from the medium opens scMUC, while addition of $\mathrm{Ca}^{2+}$ closes it. This is an efficient signaling system that controls ATP production during different events such as the cell cycle or mating. At short incubation times, the effects of $\mathrm{Ca}^{2+}$ and EGTA are fully reversible. To test the reversibility after several minutes when using $\mathrm{Ca}^{2+}$ as an effector, mitochondria were incubated in the presence of EGTA for increasing incubation times and then $\mathrm{Ca}^{2+}$ was added to promote closing. Measurements of $\Delta \Psi$ and mitochondrial swelling were conducted to evaluate PT reversibility. Yeast mitochondria were incubated in the presence of EGTA for 15, 30 seconds and 1, 2 and 4 min. Then $\mathrm{Ca}^{2+}$ was added and the effects were evaluated. At low Pi and in the presence of EGTA, the addition of $\mathrm{K}^{+}$led to a decrease of $\Delta \Psi$. Then, the addition of $\mathrm{Ca}^{2+}$ at the indicated times pro- 
moted a recovery of the $\Delta \Psi$. This was observed at all the incubation times tested (FIG $5 \mathrm{~A} \& \mathrm{~B})$. When swelling of mitochondria was tested at low Pi and in the presence of EG$\mathrm{TA}, \mathrm{K}^{+}$promoted swelling, which was reverted by $\mathrm{Ca}^{2+}$ addition at all the incubation times tested. Thus, addition of $\mathrm{Ca}^{2+}$ led to full recovery of $\Delta \Psi$ (FIG 5A) and to reversal of swelling (FIG 5B) indicating that scMUC closed after long incubation times in the open state. Reversal was even more pronounced than the one obtained using ATP/ADP additions.

Figure 5. Effect of the addition of $\mathrm{Ca}^{2+}$ on the mitochondrial transmembrane potential and mitochondrial swelling

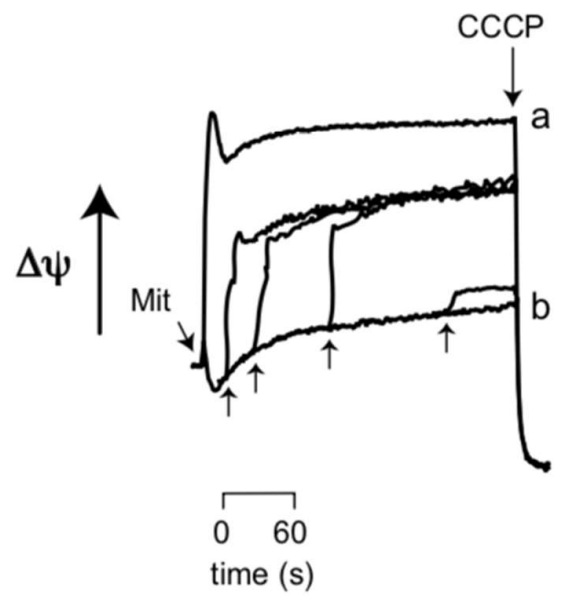

(A)

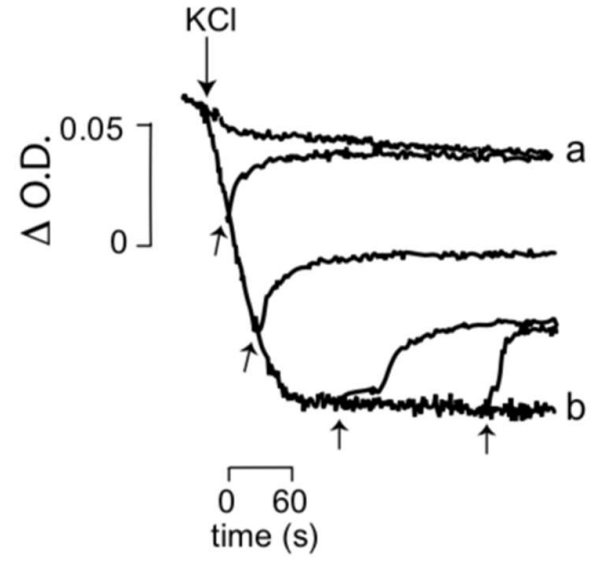

(B)

Figure 5. Effect of the addition of $\mathrm{Ca}^{2+}$ on the mitochondrial transmembrane potential and mitochondrial swelling. A: Reaction mixture as in Fig. 2. $\mathrm{Ca}^{2+} 600 \mu \mathrm{M}$ additions (upward arrows) were at different times: $30 \mathrm{sec}, 1,2$ and $4 \mathrm{~min}$. B: Reaction mixture as in Fig. 3. Where $0.1 \mathrm{M} \mathrm{KCl}$. Additions of $600 \mu \mathrm{M} \mathrm{Ca}^{2+}$ were at: $30 \mathrm{sec}, 1,2$ and 4 min (upward arrows).

\section{Discussion}

S. cerevisiae exhibits a high rate of electron flow through the RC that is further accelerated in the presence of glucose [40]. This is reminiscent of the Crabtree effect originally reported by Otto Warburg in cancer cells [40]. Both in cancer cells and in yeast, energy for growth is provided by anaerobic glycolysis [41]. It has been proposed that in tumor cells, the uncoupled, fast rate of $\mathrm{O}_{2}$ consumption is needed to inhibit production of ROS, helping the cells to survive $[42,43]$. In contrast, in S. cerevisiae this does not seem to be the case as these cells deplete $\mathrm{O}_{2}$ thriving in anoxic environments.

The high rate of $\mathrm{O}_{2}$ consumption by $S$. cerevisiae is slightly uncoupled due to different factors. First, yeast mitochondria do not have a proton-pumping respiratory Complex I, as it is substituted by three alternative NADH dehydrogenases (ND2) [15]. ND2s catalyze a much faster electron transfer than Complex I, which has a slow, complicated catalytic cycle. ND2s do not contribute to the protonmotive force so the ADP/O is lower than in other (mammalian) mitochondria. In addition, they express $s_{c} \mathrm{MUC}, \mathrm{a}$ 
channel regulated by the energy charge [19]. Indeed in our hands, ADP closed the scMUC while ATP opened it. So a high energy charge uncouples OxPhos releasing $\mathrm{O}_{2}$ consumption, while a low energy charge couples OxPhos to optimize the synthesis of ATP [8].

A system to deplete $\mathrm{O}_{2}$ would confer yeast with an advantage over other organisms as these cells thrive in hypoxic/anoxic conditions. The reversibility of the permeability transition by the alternate addition and withdrawal of calcium has been documented [18]. Mitochondria from yeast do not have a calcium transporter and the slow uptake of this ion may have been a factor in our results [44]. In contrast mammalian cells move $\mathrm{Ca}^{2+}$ through a mitochondrial uniporter (MCU) controlling respiration, mitophagy/ autophagy, and mitochondrial apoptosis [45].

Unlike mammals, in S. cerevisiae cytoplasmic $\mathrm{Ca}^{2+}$ transients may last up to 60 minutes, signaling for processes such as mating or during the cell cycle. Perhaps these are the times when the highest mitochondrial ATP production is needed. In addition, external events such as, alteration in osmolarity or the recovery response from starvation once a substrate becomes available [46]. When a haploid type a cell detects alpha-pheromone, it needs to form a large projection designed to reach for an alpha cell nearby. Then, both cells mate and become a diploid, a process that probably requires a large amount on energy. In these circumstances no apoptotic mechanisms are triggered [47]. Instead, $\mathrm{Ca}^{2+}$ probably enhances OxPhos. Indeed, $\mathrm{Ca}^{2+}$ efficiently closed $s_{c} \mathrm{MUC}$ increasing $\Delta \Psi$ and reversing mitochondrial swelling. Thus, in S. cerevisiae, PT was alternatively evoked by ATP and reversed by ADP plus Pi. i.e. a decrease in energy charge optimizes OxPhos. In each case, OxPhos is stimulated by either low energy charge (an decrease in ATP/ADP) or an increase in $\mathrm{Ca}^{2+}$, which indicates that a large quantity of ATP will be needed.

In mammals, mPTP is related to a stress response. It is interesting to note that training confers heart mPTPs with both, the ability to open and thus avoid overproducing ROS and to close after an ischemic event, re-coupling OxPhos and helping the cell to survive an ischemic event $[24,48]$. In this regard, it has been observed that during cardiac stress situations, intermittent episodes of reperfusion allow the cell to recover ATP pools and avoid ROS overproduction, greatly improving survival probabilities. This procedure is termed conditioning $[49,50]$.

In S. cerevisiae, the opening of the ${ }_{s} \mathrm{MUC}$ probably does not trigger apoptosis [10] but instead seems to help deplete $\mathrm{O}_{2}$. Most likely, the widely different roles that mPTP and ScMUC have is a classical example of exaptation, where two similar structures with similar mechanisms of action serve different functions in their respective organisms.[5154] This is further complicated by the fact that the molecules constituting each channel are still undefined.

\section{Materials and Methods}

Reagents: All chemicals were analytic grade. Mannitol, MES hydrate, D- Galactose, TEA (triethanolamine), ADP, ATP, safranine-O, oligomycin were from Sigma Chem Co. Bovine serum albumin from Probulmin TM. Bacto-peptone and yeast extract from MCD LAB. $\mathrm{H}_{3} \mathrm{PO}_{4}, \mathrm{KCl}$ and $\mathrm{MgCl}_{2}$ were from J.T. Baker.

Yeast strain. Experiments were conducted using either a commercial strain of baker's yeast Saccharomyces cerevisiae ("La Azteca, S. A." Mexico City) was used [26] or a

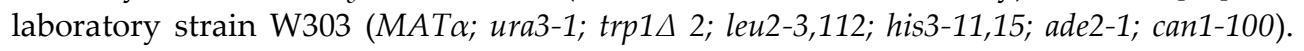
Results were similar foe each strain. Each strain was maintained in YPD (1\% yeast extract, $2 \%$ bacto-peptone, $2 \%$ glucose and $2 \%$ bacto-agar) plate cultures. Cells were grown as follows: pre-cultures were prepared immersing a loophole of yeast into $100 \mathrm{~mL}$ YPD and incubated for 24 hours under continuous agitation in an orbital shaker (New Brunswick Scientific) at $250 \mathrm{rpm}$ in a constant-temperature room $\left(30^{\circ} \mathrm{C}\right)$. Then each 100 
mL flask was used to inoculate $900 \mathrm{~mL}$ YPGal of (1\% yeast extract, $2 \%$ bacto-peptone and $2 \%$ galactose). Incubation was continued for $48 \mathrm{~h}$ under the same conditions.

Isolation of yeast mitochondria. After incubation, yeast was centrifuged (5000 xg for $5 \mathrm{~min}$, twice) and resuspended in $0.6 \mathrm{M}$ mannitol, $5 \mathrm{mM}$ MES, $0.1 \%$ bovine serum albumin, pH 6.8 (TEA). The cell suspension was mixed with $50 \%$ (v/v) of $0.5 \mathrm{~mm}$ diameter glass beads and disrupted in a Bead Beater and mitochondria were isolated from the homogenate by differential centrifugation as previously described [55]. The concentration of mitochondrial protein was determined by Biuret [56].In all assays $0.5 \mathrm{mg}$ mitochondrial protein/mL. In all experiments the mitochondrial sample was pre-incubated for $5 \mathrm{~min}$ with oligomycin (4 $\mu \mathrm{g}$ per mg protein).

Oxygen consumption. Experiments were conducted using a Clark electrode (Oximeter model 782, Warner/Strathkelvin Instruments) in a water-jacketed chamber. Temperature was kept at $30^{\circ} \mathrm{C}$ using a water bath (PolyScience $7 \mathrm{~L}$ ). Total volume $1.0 \mathrm{~mL}$. The reaction mixture for yeast mitochondria was $0.6 \mathrm{M}$ mannitol, $5 \mathrm{mM}$ MES (TEA), pH 6.8 plus $0.1 \mathrm{M} \mathrm{KCl}, 0.5 \mathrm{mM} \mathrm{MgCl} 2$ and $2 \mu \mathrm{L} / \mathrm{mL}$ ethanol. Where indicated, oligomycin 4 $\mu \mathrm{g} / \mathrm{mg}$ prot. preincubating for $5 \mathrm{~min}$.

Transmembrane potential. $\Delta \mathrm{Y}$ was determined as described by Akerman and Wikström [57], following the changes in absorbance of safranine-O at 511-533 in a double beam Aminco-Ollis spectrophotometer in dual mode. The concentrations of ATP, ADP, $\mathrm{Ca}^{2+}$ and EGTA used are indicated under each figure. At the end of each trace, the collapse in $\Delta \Psi$ was induced by addition of $6 \mu \mathrm{M}$ CCCP.

Mitochondrial swelling. Experiments were performed by following the decrease in absorbance of a mitochondrial suspension in a DW2000 Ollis/Aminco spectrophotometer in split mode at a wavelength of $540 \mathrm{~nm}$ [37].

\begin{abstract}
Author Contributions: Conceptualization, S Uribe-Carvajal and N Chiquete-Félix.; methodology ; software; formal analysis, .; investigation, P Castañeda Tamez, N Chiquete-Félix, L Morales-García and C Ricardez-García; writing-original draft preparation,. L Morales-García and C Ricardez-García ; writing-review and editing, L Morales-García, C Ricardez-García and S Uribe-Carvajal .; supervision, S Uribe-Carvajal. All authors have read and agreed to the published version of the manuscript.". All have contributed substantially to the work reported.
\end{abstract}

Acknowledgments: Partially funded by DGAPA/PAPIT/UNAM Project IN208821. LMG is a PhD student at the Ciencias Biomédicas Program, Universidad Nacional Autónoma de México ( UNAM ). PCT is a PhD student at the Biochemistry Program al UNAM, CRG is a MsC student at the Biochemistry program at UNAM. PCT, LMG and CRG are CONACyT fellows.

Nicholls, D. G. S. J. . Bioenergetics 4; 2013.

Barker Murray M; Stacey D B E; Stroud, A. K.; Peter Mitchell. Coupling of Phosphorylation to Electron and Hydrogen Transfer by a Chemiosmotic Type of Mechanism; 1944; Vol. 38.

Berry, B. J.; Trewin, A. J.; Amitrano, A. M.; Kim, M.; Wojtovich, A. P. Use the Protonmotive Force: Mitochondrial Uncoupling and Reactive Oxygen Species HHS Public Access. J Mol Biol 2018, 430 (21), 3873-3891. https://doi.org/10.1016/j.jmb.2018.03.025.

Nolfi-Donegan, D.; Braganza, A.; Shiva, S. Mitochondrial Electron Transport Chain: Oxidative Phosphorylation, Oxidant Production, and Methods of Measurement. Redox Biology. Elsevier B.V. October 1, 2020. https://doi.org/10.1016/j.redox.2020.101674. 
5 Quinlan, C. L.; Perevoshchikova, I. V.; Hey-Mogensen, M.; Orr, A. L.; Brand, M. D. Sites of Reactive Oxygen Species Generation by Mitochondria Oxidizing Different Substrates. Redox Biology 2013, 1 (1), $304-312$. https://doi.org/10.1016/j.redox.2013.04.005.

Zhao, R. Z.; Jiang, S.; Zhang, L.; Yu, Z. Bin. Mitochondrial Electron Transport Chain, ROS Generation and Uncoupling (Review). International Journal of Molecular Medicine. Spandidos Publications 2019, pp 3-15. https://doi.org/10.3892/ijmm.2019.4188.

Li, X.; Fang, P.; Mai, J.; Choi, E. T.; Wang, H.; Yang, X.-F. Targeting Mitochondrial Reactive Oxygen Species as Novel Therapy for Inflammatory Diseases and Cancers; 2013. https://doi.org/10.1186/1756-8722-6-19.

Kadenbach, B. Intrinsic and Extrinsic Uncoupling of Oxidative Phosphorylation. Biochimica et Biophysica Acta - Bioenergetics. Elsevier June 2003, pp 77-94. https://doi.org/10.1016/S0005-2728(03)00027-6.

Demine; Renard; Arnould. Mitochondrial Uncoupling: A Key Controller of Biological Processes in Physiology and Diseases. Cells 2019, 8 (8), 795. https://doi.org/10.3390/cells8080795.

10 Guerrero-Castillo, S.; Araiza-Olivera, D.; Cabrera-Orefice, A.; Espinasa-Jaramillo, J.; Gutiérrez-Aguilar, M.; Luévano-Martínez, L. A.; Zepeda-Bastida, A.; Uribe-Carvajal, S. Physiological Uncoupling of Mitochondrial Oxidative Phosphorylation. Studies in Different Yeast Species. Journal of Bioenergetics and Biomembranes 2011, 43 (3), $323-331$. https://doi.org/10.1007/s10863-011-9356-5.

Fillingame, R. H. Coupling H+ Transport and ATP Synthesis in F1F(o)-ATP Synthases: Glimpses of Interacting Parts in a Dynamic Molecular Machine. Journal of Experimental Biology 1997, 200 (2), 217-224.

Kane, P. M. Proton Transport and PH Control in Fungi. In Advances in Experimental Medicine and Biology; Springer, Cham, 2016; Vol. 892, pp 33-68. https://doi.org/10.1007/978-3-319-25304-6_3.

Catty, P.; De Kerchove D'exaerde, A.; Goflfeau, A. The Complete Inventory of the Yeast Saccharomyces Cerevisiae P-Type Transport ATPases; 1997; Vol. 409. https://doi.org/10.1016/S0014-5793(97)00446-8.

Jouhten, P.; Rintala, E.; Huuskonen, A.; Tamminen, A.; Toivari, M.; Wiebe, M.; Ruohonen, L.; Penttilä, M.; Maaheimo, H. Oxygen Dependence of Metabolic Fluxes and Energy Generation of Saccharomyces Cerevisiae CEN.PK113-1A. 2008. https://doi.org/10.1186/1752-0509-2-60.

Vemuri, G. N.; Eiteman, M. A.; Mcewen, J. E.; Olsson, L.; Nielsen, J. Increasing NADH Oxidation Reduces Overflow Metabolism in Saccharomyces Cerevisiae; 2007; Vol. 13.

Mileykovskaya, E.; Penczek, P. A.; Fang, J.; Mallampalli, V. K. P. S.; Sparagna, G. C.; Dowhan, W. Arrangement of the Respiratory Chain Complexes in Saccharomyces Cerevisiae Supercomplex III2IV2 Revealed by Single Particle Cryo-Electron Microscopy. Journal of Biological Chemistry 2012, $287 \quad$ (27), 23095-23103. https://doi.org/10.1074/jbc.M112.367888.

Uribe-Carvajal, S.; Luévano-Martínez, L. A.; Guerrero-Castillo, S.; Cabrera-Orefice, A.; Corona-de-la-Peña, N. A.; Gutiérrez-Aguilar, M. Mitochondrial Unselective Channels throughout the Eukaryotic Domain. Mitochondrion. May 6, 2011, pp 382-390. https://doi.org/10.1016/j.mito.2011.02.004.

Cabrera-Orefice, A.; Ibarra-García-Padilla, R.; Maldonado-Guzmán, R.; Guerrero-Castillo, S.; Luévano-Martínez, L. A.; Pérez-Vázquez, V.; Gutiérrez-Aguilar, M.; Uribe-Carvajal, S. The Saccharomyces Cerevisiae Mitochondrial Unselective Channel Behaves as a Physiological Uncoupling System Regulated by Ca2+, Mg2+, Phosphate and ATP. Journal of Bioenergetics and Biomembranes 2015, 47 (6), 477-491. https://doi.org/10.1007/s10863-015-9632-x. 
Pérez-Vázquez, V.; Saavedra-Molina, A.; Uribe, S. In Saccharomyces Cerevisiae, Cations Control the Fate of the Energy Derived from Oxidative Metabolism Through the Opening and Closing of the Yeast Mitochondrial Unselective Channel. Journal of Bioenergetics and Biomembranes 2003, 35 (3), 231-241. https://doi.org/10.1023/A:1024659615022.

Manon, S.; Roucou, X.; Guérin, M.; Rigoulet, M.; Guérin, B. Minireview: Characterization of the Yeast Mitochondria Unselective Channel: A Counterpart to the Mammalian Permeability Transition Pore? Journal of Bioenergetics and Biomembranes. 1998, pp 419-429. https://doi.org/10.1023/A:1020533928491.

Hüser, J.; Rechenmacher, C. E.; Blatter, L. A. Imaging the Permeability Pore Transition in Single Mitochondria. Biophysical Journal 1998, 74 (4), 2129-2137. https://doi.org/10.1016/S0006-3495(98)77920-2.

Haworth, R. A.; Hunter, D. R. The Ca2+-Induced Membrane Transition in Mitochondria. II. Nature of the Ca2+ Trigger Site. Archives of Biochemistry and Biophysics 1979, 195 (2), 460-467. https://doi.org/10.1016/0003-9861(79)90372-2.

Correa, F.; Soto, V.; Zazueta, C. Mitochondrial Permeability Transition Relevance for Apoptotic Triggering in the Post-Ischemic Heart. International Journal of Biochemistry and Cell Biology 2007, 39 (4), 787-798. https://doi.org/10.1016/j.biocel.2007.01.013.

Correa, F.; García, N.; Robles, C.; Martínez-Abundis, E.; Zazueta, C. Relationship between Oxidative Stress and Mitochondrial Function in the Post-Conditioned Heart. Journal of Bioenergetics and Biomembranes 2008, 40 (6), 599-606. https://doi.org/10.1007/s10863-008-9186-2.

Zoratti, M.; Szabò, I. The Mitochondrial Permeability Transition. BBA - Reviews on Biomembranes 1995, 1241 (2), $139-176$. https://doi.org/10.1016/0304-4157(95)00003-A.

Hurst, S.; Hoek, J.; Sheu, S.-S. MINI-REVIEW Mitochondrial Ca 2+ and Regulation of the Permeability Transition Pore. https://doi.org/10.1007/s10863-016-9672-x.

Crompton, M.; Costi, A.; Hayat, L. Evidence for the Presence of a Reversible Ca2+-Dependent Pore Activated by Oxidative Stress in Heart Mitochondria; 1987; Vol. 245.

Hunter, D. R.; Haworth, R. A. The Ca2+-Induced Membrane Transition in Mitochondria. I. The Protective Mechanisms. Archives of Biochemistry and Biophysics 1979, 195 (2), 453-459. https://doi.org/10.1016/0003-9861(79)90371-0.

Halestrap, A. P. Mitochondrial Permeability: Dual Role for the ADP/ATP Translocator? Nature. Nature 2004. https://doi.org/10.1038/nature02816.

Racay, P. Effect of Magnesium on Calcium-Induced Depolarisation of Mitochondrial Transmembrane Potential. Cell Biology International 2008, 32 (1), 136-145. https://doi.org/10.1016/j.cellbi.2007.08.024.

Porter, G. A.; Beutner, G. Cyclophilin D, Somehow a Master Regulator of Mitochondrial Function. Biomolecules. MDPI AG December 1, 2018. https://doi.org/10.3390/biom8040176.

Lu, X.; Kwong, J. Q.; Molkentin, J. D.; Bers, D. M. Individual Cardiac Mitochondria Undergo Rare Transient Permeability Transition Pore Openings. Circulation Research 2016, 118 (5), 834-841. https://doi.org/10.1161/CIRCRESAHA.115.308093.

Boyman, L.; Coleman, A. K.; Zhao, G.; Wescott, A. P.; Joca, H. C.; Greiser, B. M.; Karbowski, M.; Ward, C. W.; Lederer, W. J. Dynamics of the Mitochondrial Permeability Transition Pore: Transient and Permanent Opening Events. Archives of Biochemistry and Biophysics 2019, 666, 31-39. https://doi.org/10.1016/j.abb.2019.03.016.

Belosludtsev, K. N.; Dubinin, M. V; Talanov, E. Y.; Starinets, V. S.; Tenkov, K. S.; Zakharova, N. M.; Belosludtseva, N. V. Transport of Ca 2+ and Ca 2+-Dependent Permeability Transition in the Liver and Heart Mitochondria of Rats with Different Tolerance to Acute Hypoxia. https://doi.org/10.3390/biom10010114. 
Pérez-Vázquez, V.; Saavedra-Molina, A.; Uribe, S. In Saccharomyces Cerevisiae, Cations Control the Fate of the Energy Derived from Oxidative Metabolism Through the Opening and Closing of the Yeast Mitochondrial Unselective Channel. Journal of Bioenergetics and Biomembranes 2003, 35 (3), 231-241. https://doi.org/10.1023/A:1024659615022.

Bradshaw, P. C.; Pfeiffer, D. R. Loss of NAD(H) from Swollen Yeast Mitochondria. BMC Biochemistry $2006,7$. https://doi.org/10.1186/1471-2091-7-3.

Halestrap, A. P.; Davidson, A. M. Inhibition of Ca2+-Induced Large-Amplitude Swelling of Liver and Heart Mitochondria by Cyclosporin Is Probably Caused by the Inhibitor Binding to Mitochondrial-Matrix Peptidyl-Prolyl Cis-Trans Isomerase and Preventing It Interacting with the Adenine Nucleotide Translocase; 1990; Vol. 268.

Solesio, M. E.; Demirkhanyan, L.; Zakharian, E.; Pavlov, E. V. Contribution of Inorganic Polyphosphate towards Regulation of Mitochondrial Free Calcium. Biochimica et Biophysica Acta - General Subjects 2016, 1860 (6), 1317-1325. https://doi.org/10.1016/j.bbagen.2016.03.020.

Li, W.; Zhang, C.; Sun, X. Mitochondrial Ca2+ Retention Capacity Assay and Ca2+-Triggered Mitochondrial Swelling Assay. Journal of Visualized Experiments 2018, 2018 (135), 1-7. https://doi.org/10.3791/56236.

(1)

Rodriguez-Enriquez, S.; Marín Hernández, A.; Uribe-Carvajal, S.; Devin, A.; Bouchez, C. L.; Hammad, N.; Cuvellier, S.; Ransac, S.; Rigoulet, M. The Warburg Effect in Yeast: Repression of Mitochondrial Metabolism Is Not a Prerequisite to Promote Cell Proliferation. Frontiers in Oncology I www.frontiersin.org 2020, 1, 1333. https://doi.org/10.3389/fonc.2020.01333.

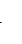

Liberti, M. V; Locasale, J. W. The Warburg Effect: How Does It Benefit Cancer Cells? (Vol 41, Pg 211, 2016). Trends in Biochemical Sciences 2016, 41 (3, SI), 287. https://doi.org/10.1016/j.tibs.2015.12.001.The.

Cortassa, S.; O’Rourke, B.; Aon, M. A. Redox-Optimized ROS Balance and the Relationship between Mitochondrial Respiration and ROS. Biochimica et Biophysica Acta - Bioenergetics 2014, 1837 (2), $287-295$. https://doi.org/10.1016/j.bbabio.2013.11.007.

Kembro, J. M.; Aon, M. A.; Winslow, R. L.; O’Rourke, B.; Cortassa, S. Integrating Mitochondrial Energetics, Redox and ROS Metabolic Networks: A Two-Compartment Model. Biophysical Journal 2013, $104 \quad(2)$, 332-343. https://doi.org/10.1016/j.bpj.2012.11.3808.

Kovaćs-Bogdán, E.; Sancak, Y.; Kamer, K. J.; Plovanich, M.; Jambhekar, A.; Huber, R. J.; Myre, M. A.; Blower, M. D.; Mootha, V. K. Reconstitution of the Mitochondrial Calcium Uniporter in Yeast. Proceedings of the National Academy of Sciences of the United States of America 2014, 111 (24), 8985-8990. https://doi.org/10.1073/pnas.1400514111.

Alevriadou, B. R.; Patel, A.; Noble, M.; Ghosh, S.; Gohil, V. M.; Stathopulos, P. B.; Madesh, M. Molecular Nature and Physiological Role of the Mitochondrial Calcium Uniporter Channel. American Journal of Physiology - Cell Physiology 2021, 320 (4), C465-C482. https://doi.org/10.1152/ajpcell.00502.2020.

Busti, S.; Mapelli, V.; Tripodi, F.; Sanvito, R.; Magni, F.; Coccetti, P.; Rocchetti, M.; Nielsen, J.; Alberghina, L.; Vanoni, M. Respiratory Metabolism and Calorie Restriction Relieve Persistent Endoplasmic Reticulum Stress Induced by Calcium Shortage in Yeast. Scientific Reports 2016, 6. https://doi.org/10.1038/srep27942.

Eisenberg, T.; Büttner, S.; Kroemer, G.; Madeo, F. The Mitochondrial Pathway in Yeast Apoptosis. Apoptosis. May 2007, pp 1011-1023. https://doi.org/10.1007/s10495-007-0758-0.

Panel, M.; Ruiz, I.; Brillet, R.; Lafdil, F.; Teixeira-Clerc, F.; Nguyen, C. T.; Calderaro, J.; Gelin, M.; Allemand, F.; Guichou, J. F.; Ghaleh, B.; Ahmed-Belkacem, A.; Morin, D.; Pawlotsky, J. M. Small-Molecule Inhibitors of Cyclophilins Block Opening of the Mitochondrial Permeability Transition Pore and Protect Mice From Hepatic Ischemia/Reperfusion Injury. Gastroenterology 2019, 157 (5), 1368-1382. https://doi.org/10.1053/j.gastro.2019.07.026. 
Ramírez-Camacho, I.; Correa, F.; El Hafidi, M.; Silva-Palacios, A.; Ostolga-Chavarría, M.; Esparza-Perusquía, M.; Olvera-Sánchez, S.; Flores-Herrera, O.; Zazueta, C. Cardioprotective Strategies Preserve the Stability of Respiratory Chain Supercomplexes and Reduce Oxidative Stress in Reperfused Ischemic Hearts. Free Radical Biology and Medicine 2018, 129, 407-417. https://doi.org/10.1016/j.freeradbiomed.2018.09.047.

Lesnefsky, E. J.; Hoppel, C. L. Ischemia-Reperfusion Injury in the Aged Heart: Role of Mitochondria. Archives of Biochemistry and Biophysics. Academic Press Inc. December 15, 2003, pp 287-297. https://doi.org/10.1016/j.abb.2003.09.046.

Brosius, J. Exaptation at the Molecular Genetic Level. Science China Life Sciences. Science in China Press April 1, 2019, pp 437-452. https://doi.org/10.1007/s11427-018-9447-8.

Bowen, N.; Jordan, I. Exaptation of Protein Coding Sequences from Transposable Elements. Genome Dynamics. Genome Dyn 2007, pp 147-162. https://doi.org/10.1159/000107609. Barsukova, A. G.; Bourdette, D.; Forte, M. Mitochondrial Calcium and Its Regulation in Neurodegeneration Induced by Oxidative Stress. European Journal of Neuroscience 2011, 34 (3), 437-447. https://doi.org/10.1111/j.1460-9568.2011.07760.x. Mitochondrial Permeability Transition Pore (PTP) - An Example of Multiple Molecular Exaptation? Biochimica et Biophysica Acta - Bioenergetics. 2012, pp 2072-2086. https://doi.org/10.1016/j.bbabio.2012.06.620.

Peña, A.; Piña, M. Z.; Escamilla, E.; Piña, E. A Novel Method for the Rapid Preparation of Coupled Yeast Mitochondria. FEBS Letters 1977, 80 (1), 209-213. https://doi.org/10.1016/0014-5793(77)80441-9.

Gornall, A. G.; Bardawill, C. J.; David, M. M. Determination of Serum Proteins by Means of the Biuret Reaction. The Journal of biological chemistry 1949, 177 (2), 751-766.

Åkerman, K. E. O.; Wikström, M. K. F. Safranine as a Probe of the Mitochondrial Membrane Potential. FEBS Letters 1976, 68 (2), 191-197. https://doi.org/10.1016/0014-5793(76)80434-6. 